\title{
Porous Silicon Biosensors
}

\author{
M. B. de la Mora, M. Ocampo, R. Doti, J. E. Lugo and \\ J. Faubert
}

Additional information is available at the end of the chapter

http://dx.doi.org/10.5772/52975

\section{Introduction}

There are a vast number of applications for biosensors ranging from medical monitoring and control, to release of drugs [1], and biosecurity [2]. The goblal market for biosensors in 2012 is estimated to reach 8.5 billion USD and projected to reach 16.8 billion by 2018 [3]. Porous silicon ( $\mathrm{p}-\mathrm{Si}$ ) offers several advantages for its use as a biosensor such as a large specific surface area (of the order of $500 \mathrm{~m}^{2} \mathrm{~cm}^{-3}$ ) [4], visible luminescence at room temperature [5] and biocompatibility [6]. The p-Si was accidentally discovered when, in 1956 at the U.S. Bell Laboratories, Arthur Uhlir Jr. and Ingeborg Uhlir observed a red-green film formed on the wafer surface while trying a new technique for polishing silicon (Si) crystalline wafers. At the time however, it was not considered an interesting material. But when Leigh Canham in 1990 [5] discovered its visible luminescence properties, researchers started studying its nonlinear optical, electric and mechanical properties. These academic and technological efforts have permitted the fabrication of uniform porous layers with diameters as small as one nanometer, permitting an enormous inner surface density, which is useful for biosensing applications. Several techniques exist to form this structure from a pure silicon crystalline wafer. The most popular is the electrochemical etching of crystalline silicon wafers (c-Si) [5]. Anodization begins when a constant current is applied between the c-Si wafer and the electrolyte by means of an electronic circuit controlling the anodization process [6].

Generally, p-Si is fabricated as shown in figure 1. We have a c-Si wafer (single crystalline) with the top face in contact with a hydrofluoric acid solution (HF) and where an immersed platinum electrode is placed at certain distance and parallel to the wafer. In the bottom face of the wafer we find a flat metallic electrode that is in close electric contact. Between the two electrodes there is a controlled voltage supply with its negative pole connected to the platinum immersed electrode. A current is established from the anodic electrode (back of the wafer) and 
the catodic electrode (platinum immersed). Modulating four variables: the intensity and interval of application of this current, the HF solution concentration, and the concentration and type of dopant previously applied to the c-Si wafer (type-n, type-p, or highly doped: type- $\mathrm{p}^{+}$and type $\mathrm{n}^{+}$) it is then possible to control the porous size and p-Si layer geometrical parameters, as well as the number of layers. Dopant refers to a different element atom that replaces a percentage of the Si atom inside the wafer and that is crystallographically compatible with it, but that presents an electron in excess (type $\mathrm{n}$ ) or an electron lack (type $\mathrm{p}$ ). This introduces a number of properties that modify the material behavior when an electric field is applied, mainly the resistivity, that will influence the etching process performance.

The electric current oxidizes the surface silicon atoms permitting a fluoride ion (formed in the HF solution because of the electrical current) attack on them generating the pores. By using this electrochemical methodology it is also possible to create multilayer structures by alternating different current densities. For instance, if we start making the first layer with a current density J1 then the final porosity (and the refractive index) is going to be approximately determined by this current density. The electrochemical reaction time determines the thickness. By switching the current density to a different value J2, the reaction mainly continues at the crystalline silicon interface, leaving an almost intact first layer. Then the second layer will have a different refractive index and thickness (if we readjust the reaction time).

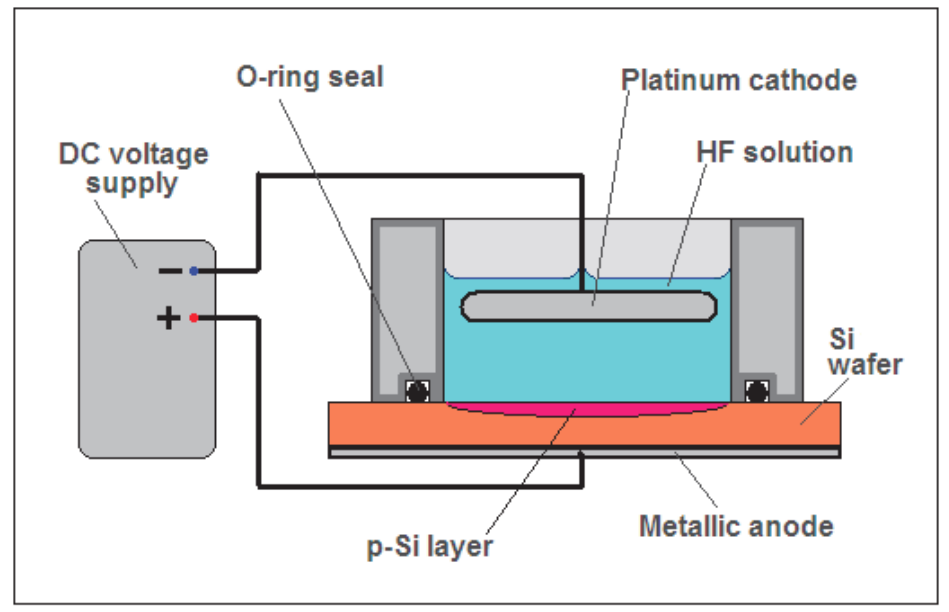

Figure 1. Experimental setup for porous silicon fabrication.

The figure 2 shows two structures that we fabricated from electrochemical etching of porous silicon. A luminescent monolayer made from p-type silicon wafers with a resistivity of 1-2 $\mathrm{Ohm} / \mathrm{cm}$ (Fig. $2 \mathrm{a}$ ), and a multilayer prepared from p-type silicon wafers with a resistivity of $0.001-0.005 \mathrm{Ohm} / \mathrm{cm}$ (Fig. 2 b). Notice that by changing the dopant concentration, which is relate to the electrical resistivity used, the characterisitics of the p-Si structures differ. High 
resistivity crystalline silicon wafers give us higher porosities and small nanowires related to a given luminescent behavior. In turn, low resistivity allows us to achieve multilayers structures.

After the electrochemical etching stage, the surface of p-Si is hydrogen-terminated; this permits to immobilize large amounts of biomolecules [7]. It is possible to control several parameters of p-Si such as; pore size and consequently the refractive index, thickness, morphology, etc. by modifying the anodization conditions $[6,11]$. Porosity can be measured by gravimetrical means. That is, the original crystalline silicon wafer is weighed first, then p-Si is formed and the wafer is weighed again, finally the p-Si layer is removed by adding $\mathrm{KOH}$ (Potassium hydroxide) and the wafer is weighed once more. With these three measurements is possible to determine the porosity. To measure the thickness, SEM (scanning electronic microscopy) techniques are normally used giving the best resolution and accuracy. Refractive index is usually determined by optical interference methods, where the refractive index can be estimated by taking adjacent maxima or minima from interference fringes coming from the p-Si sample.

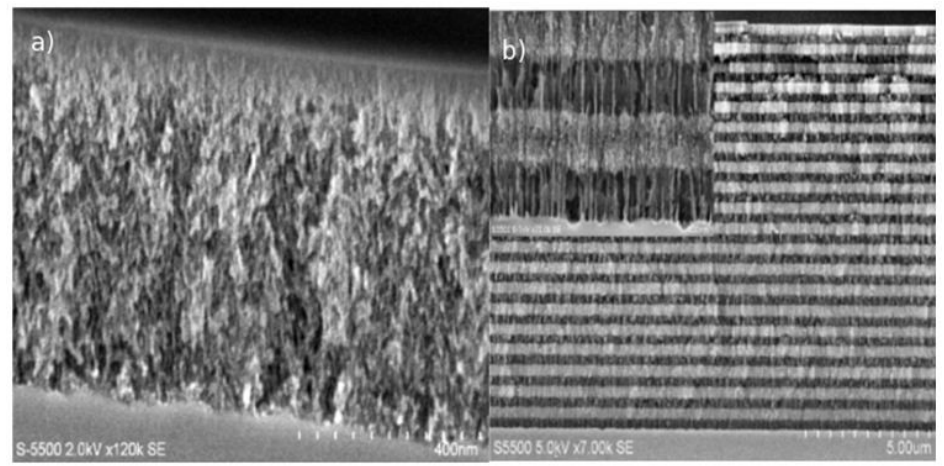

Figure 2. Crossectional SEM images of porous silicon nanostructures. A luminescent monolayer (a) and a multilayer (b). These strucutures were prepared at CIE-UNAM porous silicon laboratory.

There are other methods for obtaining p-Si such as the photoelectrochemical [5], the chemical vapour etching [8], the metal-assisted etching [9], and the 'stain etching' procedure [10]. The last two techniques mentioned do not require an electrical bias. In the stain etching procedure the power supply action is replaced by the chemical oxidant action of nitric acid. The reaction control is performed trough the addition of other additives. The results are less homogeneous than for the first process described, but they still permit to have the material quality compatible with several applications. As an example in figure 3 we show SEM images of a p-SI monolayer obtained by metal assisted etching of gold nanostructures and subsequent chemical attack of an $\mathrm{HF} / \mathrm{H}_{2} \mathrm{O}_{2}$ electrolyte. 


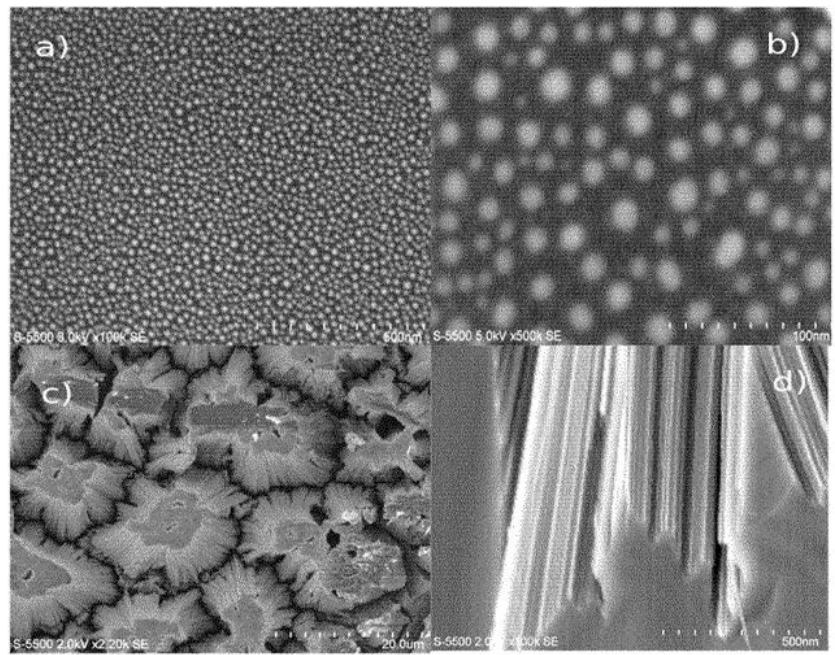

Figure 3. SEM images of gold nanostructures $(a, b)$ used to fabricated a porous silicon monolayer. We show the surface (c) and the cross sectional (d) images. These structures were prepared at CIE-UNAM porous silicon laboratory.

The p-Si material can be prepared either in powder or wafer permitting to elaborate devices that can be dispersed in a given medium or reused [12]. Furthermore $\mathrm{p}$-Si is a material that allows the fabrication of high quality photonic crystals [13] by applying the method described before to obtain multilayers structures. Such characteristics therefore allow several biosensing approaches usign this porous material [1].

\section{Porous silicon biosensors: construction and transduction principles}

The aim of a biosensor is to produce either discrete or continuous signals, which are proportional to a single analyte or a related group of analytes [14]. Because of its particular properties, the p-Si can be used as a transducer to convert this analytes into an optical or electrical signal [1]. Its large surface area enables an effective capture of the biological analytes although such a large surface area also implies high reactivity with the enviroment. This can cause the degradation of the biosensor and/or possible false positives. For this reason, stabilization of the p-Si surface via an appropriate surface chemistry is a required step for obtaining a succesful biosensor [15]. The surface chemistry should be designed in such a way as to obtain the desired effects, and yet still displaying bioactivity [16]. Also the binding affinity with the studied analytes must be taken into account $[15,17]$. Some common techniques to functionalize p-Si include: oxidation [18, 19, 20], silanization [1, 15, 21, 22, 23, 24, 25], hydrosilylation of alkenes and alkynes [27, 28], radiation [29], and other chemical approaches [15, 16].

A proper pore-size distribution helps to achieve an efficient biosensor; $\mathrm{p}$-Si fabricate from $\mathrm{p}+$ and $\mathrm{n}+$-type silicon substrates is mesoporous, and suitable for immobilisation of biomacro- 
molecules, while p-Si from p-type substrates, whose pore diameter is of the order of a few $\mathrm{nm}$, is suitable only for very small molecules [12]. Macroporous p-Si from n-type substrates may accommodate larger molecules [12].

Once the appropriate chemical functionalization and porous distribution size is obtained, the challenge then becomes transducing the recognition of the biological analytes into a measurable signal. The requirements for efficient transduction are precision (same response to the same stimuli: repeatability) and accuracy (indicating magnitude value as close as possible to the real magnitude of the stimulus to be sensed: minimum absolute error spread) [30].

In general, the most common transduction techniques include piezoresistance, piezoelectricity, capacitive, resistive, tunneling, thermoelectricity, optical and radiation-based techniques, and electrochemical methods [31]. In the case of p-Si biosensors the most frequently used tecniques are optical and electrical/ electrochemical [1]. Here, we classified the p-Si biosensors depending on the transducing mechanism in optical and electrochemical contexts. Their characteristics and some related examples are detailed in the following sections.

\section{Types of Porous silicon biosensors}

\subsection{Optical p-Si biosensors}

Chemical or biomolecule detection can be based on changes in the optical spectral interference pattern $[22,23]$. When white light passes through the $\mathrm{p}$-Si an interference pattern is observed, this effect is called a Fabry-Perot fringe pattern, the binding of molecules induces changes in this pattern which are relate to a change in the refractive index of the p-Si [22]. This change is shown by a shift of the fringe pattern that can be quantified [22]. The effect depends on the refractive index value of the analized solution but also on how it penetrates into the pores [11]. The simplest kind of such p-Si biosensors is made of mono and doublelayer films [1]. Some biological systems studied with these biosensors are: DNA hybridization [22, 32, 33], antibody cascading and the prototypical biotinstreptavidin interaction [22]. Using an analogous optical transduction modality it is possible to build p-Si biosensors with others complex optical structures as multilayer devices [6, 30].

These can be built up by alternating the applied current densities during the electrochemical etching generating a periodic or quasiperiodic combination of refractive indices [6]. This kind of structures offers better reflectance spectra (without side lobes) if compared with a mono or doubled-layered structure [30]. The etching parameters must be chosen to accommodate the analyte of interest whilst maximising the optical response. Some of the p-Si optical structures used in biosensing are: 1D photonic crystals [35, 36], rugate filters [37], microcavities [6, 38] and quasicrystals [39]. The use of these optical structures in biosensors allows integrability of all optical components and do not require electric contacts [11].

The photoluminescence properties of $\mathrm{p}$-Si are also useful mechanisms for developing biosensors. It is possible to associate the amount of analytes studied with the changes in the photoluminescence spectra $[1,40,41]$. For example the quenching in the photoluminescence spectra 
after DNA deposition was used to study the transduction of DNA hybridization [42]. In this case the behavior was attributed to non-radiative recombination processes [1]. In recent years a successful implementation of this type of biosensor was obtained [6, 32, 42,41] however until now this kind of biosensor is less accurate than its interferometric counterparts [1].

In a similar way the amount of an analyte of interest can be quantified by measuring the fluorescence signal intensity of a fluorescence molecule used as a marker fixed at a p-Si structure before and after an analyte is located into the p-Si [43].

We offer two comprehensive case examples to illustrate how the optical p-Si biosensors work. The first example is a sensor of a fluorescent molecule: fluorescein-5-maleimide (FM), by using a 1D photonic crystal or Bragg mirror [44]. The basic mirror was made by alternating layers of high (2.83) and low (1.65) refractive indexes, with a first layer that allows a good penetration of the active molecule into the porous structure. The surface of the first layer was functionalized by silanization with 3-mercaptopropyl)-trimethoxysilane (MPTS) to link the fluorescent molecule. The silicon mirror was fabricated in order to achieve a reflectance spectrum in a range that overlaps the fluorescent excitation of the molecule. The samples were analyzed by fluorescent spectrometry. The emission signal from fluorescent molecules was enhanced because of the p-Si mirror. That is, the p-Si structure provided a platform for high-sensitivity measurements. This biosensor uses two different detection platforms by using reflectance measurements as we show in the figure 4 and by analyzing the fluorescent spectrum as it can be observed in the figure 5 .

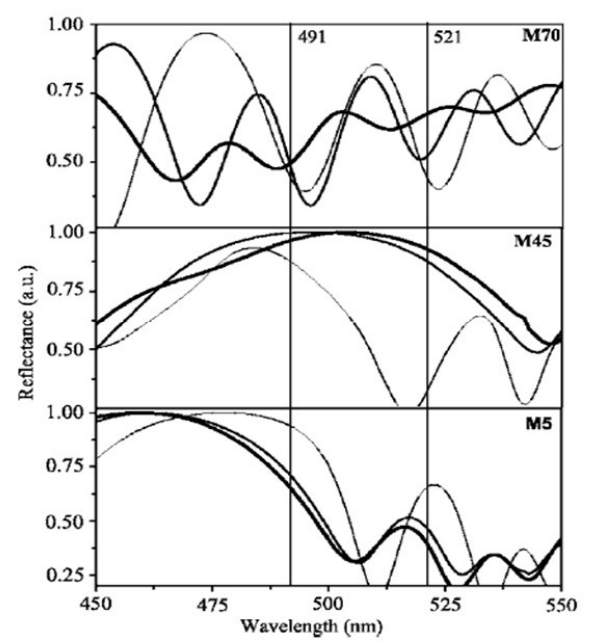

Figure 4. Reflectance spectra for freshly etched (thin line), silanized (normal line), and functionalized (thick line) mirrors. Vertical lines correspond to wavelength of excitation of $491 \mathrm{~nm}$ and emission of $521 \mathrm{~nm}$ of the FM molecule in a phosphate solution. Uncertanties in the reflectance intensity and wavelength were of $\pm 2 \%$ and $\pm 1 \mathrm{~nm}$, respectively [44]. 


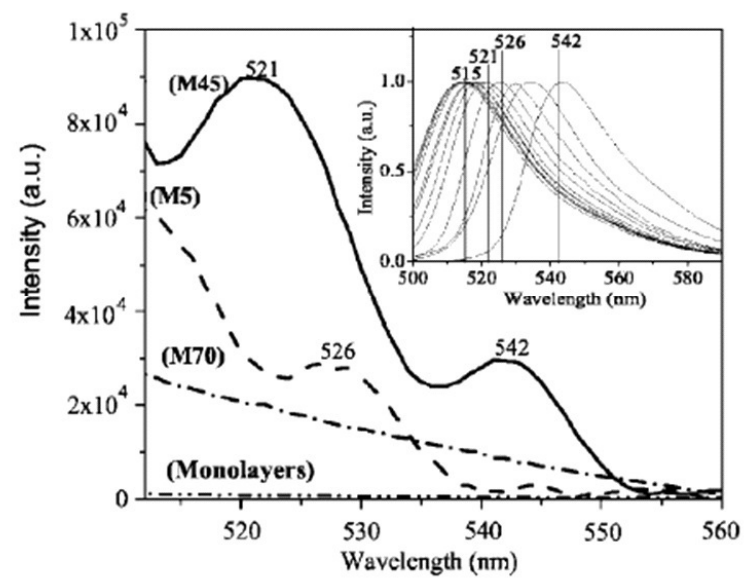

Figure 5. Fluorescence emission of FM molecules deposited on the MPTS functionalized surfaces. Monolayers correspond to sample m45 and m70 (no mirrors). Sample M45 shows the best fluorescence signal. Fluorescence emissions of FM in solution are shown in the inset for comparison; the concentrations for each spectrum from left to right, are $0.37,0.7,1.2,3.77,5.39,7.7$, and $11 \mathrm{mM}$. Uncertanties in fluorescence intensity and wavelength were of $\pm 0.1 \%$ and $\pm 1 \mathrm{~nm}$, respectively [44].

The second example is a microcavity [45]. This microcavity is formed when a luminescent p$\mathrm{Si}$ layer is inserted between two Bragg reflectors made of $\mathrm{p}$-Si. The broad luminescence band is altered and very narrow peaks are detected. The position of these peaks is extremely sensitive to a small change in refractive index, such as that obtained when a biological analyte is placed in the large internal surface of p-Si. A DNA biosensor was developed by using such an oxidized microcavity [45]. After successful silanization of the p-Si surface, DNA was immobilized into the porous surface through a careful diffusion. Finally, the DNA-attached wass exposed to its complementary strand of DNA (cDNA). A red-shift in photoluminescence is observed. Full-length viral DNA molecules were also detected with the microcavity biosensor [45vis]. The advantages of optical sensing are significantly improved when this approach is used within an integrated optics context [46].

\subsection{Electric and Electrochemical P-Si biosensors}

The highly sensitive surface of p-Si and the possibility to measure changes in its electrical properties added to its capacity to adsorb an enormous amount of different compounds, can be used for electrical biosensor applications [47,48]. These approaches consider the use of electrical contacts on the $\mathrm{p}$-Si layer made by metal deposition to measure the changes in the electrical properties such as capacitance and conductance when an analyte is attached to the p-Si layer [49]. An example of this type of biosensor is a macroporous sensor to detect DNA hybridization by characterizing the difference between the dipolar moment in p-Si layers with and without the analyte [47]. Another DNA detector of nanoporous silicon biosensor is described in reference [50]. This biosensor is an electrochemical device that transduces the 
hybridization of DNA into a chemical oxidation of guanine by $\mathrm{Ru}(\mathrm{bpy})^{2+}{ }_{3}$, the reduced form of which is then detected electrochemically.

Another effective platform to develop a p-Si biosensor is by applying electrochemical characterization. There are two main types of electrochemical transduction in biosensors: potentiometry and amperometry/voltammetry [12].

In potentiometryc biosensors the main parameter is the potential difference between the cathode and the anode in an electrochemical cell [51,52]. This difference can be transduced as an electrical signal [12]. Amperometric and voltammetric biosensors consider the redox reaction that takes place in the anodization cell when an analyted of interest is placed. In this case the analyte is immobilised and an analyte oxidation/reduction process produces a flux of electrons measured, in terms of current intensity, cross the electrodes of the electrochemical cell [12]. These biosensors are too sensitive to $\mathrm{pH}$ modifications [51].

Examples of these sensors are the potentiometric and amperometric urea sensor based on nanoporous silicon technology described by Joon-Hyung Jin et al [52]. One of the electrochemical devices consists on three thin-film electrodes patterned on p-type silicon wafer by using platinum RF sputtering and silver (Ag) evaporation. The working electrode, on which the urease is inmobilized with a polymeric conductor: polypirrole (PPy) is sensitive to urea dissolved in artificially made electrolyte solution. The reference electrode is p-Si -based Ag/ $\mathrm{AgCl}$ thin-film reference electrode (TFRE). The other is a platinum (Pt) thin-film counter electrode. In a potentiometric urea sensor, urea concentration is related to the measured potential applied between the working and reference electrode according to the Nernst equation. The other device is developed under amperometric regime. In this case the ureasecatalyzed hydrolytic reaction of urea causes current flow between the working and counter electrode and the amount of current flow is proportional to the urea concentration that represents a change of $\mathrm{pH}$, which is based on the Cottrell equation. In this study [50] it was found that urea sensitive electrodes (PSUE's) and Ag/AgCl TFRE's based on p-Si layers provides better adhesive strength between thin-films, and silicon-based electrodes. This reduces the leaching out of TFRE components and enhances the sensitivity of a sensing electrode. The presence of carbon, nitrogen and sulfur, which were attributed to the urease-doped PPy films were confirmed by EDX characterization. The p-Si-based Ag/AgCl TFRE can be recommended as an ideal non-polarizable reference electrode to determine the electrochemical cell potentials and currents of sensing electrodes. Amperometry for monitoring the urea concentrations caused by urease-catalyzed reactions is superior to a potentiometric method in that the amperometric urea sensors gives a longer linear range, higher sensitivity and shorter response times than the potentiometric urea sensors, especially at low urea concentrations.

Another very interesting application of porous silicon biosensors is for liver diagnosis [53]. Min-Jung Song et al presented a study of a biosensor array system consisting of cholesterol, bilirubin and glutamate sensors. The p-Si electrochemical system consisted of porous silicon layers formed on each working electrode that increased greatly the effective surface area. The electrodes in the sampling wells minimized a cross-interference effect to permit multiple sampling by immobilization of the enzymes using a silanization technique. The biosensor arrays tested used aqueous samples of the enzymes prepared in a $50 \mathrm{mM}$ phosphate buffer 
solution ( $\mathrm{pH}$ 8). All measurements were performed at room temperature at amperometric detection regime of each sensor was carried out using at a potential of $+0.6 \mathrm{~V} v \mathrm{vs}$. $\mathrm{Ag} / \mathrm{AgCl}$ for the biosensors of the hydrogen peroxide generated in the silanized layer where the enzymatic reactions occur. In general, normal cholesterol concentrations in the human do not exceed $200 \mathrm{mg}$ per $100 \mathrm{ml}$ [53]. Higher cholesterol concentrations are considered abnormal.

In this case, the current detected is linearly proportional to cholesterol concentrations in the range of $1 \mathrm{mM}$ to $50 \mathrm{mM}$; sensitivity was measured at approximately $0.2656 \mu \mathrm{A} / \mathrm{mM}$. The bilirubin calibration curve covers a large concentration range between $0.002 \mathrm{mM}$ and 0.020 $\mathrm{mM}$, which includes normal levels $(0.2 \sim 1.0 \mathrm{mg} / \mathrm{dl})$, and levels typical of abnormal serum bilirubin. The sensitivity of the calibration curve approximated $0.15354 \mathrm{~mA} / \mathrm{mM}$. The detection of the ratio of alanine aminotransferase (ALT) and aspartate aminotransferase (AST) that in human serum indicates an abnormal symptom of the liver is also based upon electrochemical oxidation at the Pt electrode surface. Since L-glutamate is a product of both ALT and AST reactions occurring in the buffer solution, the enzyme activities can be determined from the current changes at the L-glutamate sensor. On average, the serum ALT and AST levels measured in healthy people by optimized conventional ALT and AST assays approximates $10 \mathrm{U} / 1$ at $25^{\circ} \mathrm{C}$ and any increase in enzyme levels that exceed $100 \mathrm{U} / 1$ is taken to indicate liver disease. The sensitivity determined from the semi-logarithmic plot approximated $0.13698 \mu \mathrm{A} /(\mathrm{U} / \mathrm{l})$ for ALT over the range of $1.3 \mathrm{U} / 1$ to $250.0 \mathrm{U} / \mathrm{l}$. For AST, the sensitivity was about $0.45439 \mu \mathrm{A} /(\mathrm{U} / \mathrm{l})$ in the same concentration ranges as for ALT.

This device offers several important advantages which include

1. readout within minutes from application of microvolumes of sample,

2. reduced physical dimensions of the device,

3. relative stability of the reagents used, and

4. simple electronics applicable to the further development of a hand held device useful for point of care biomarker liver analyses.

In the following paragraph we will describe in detail an example of an electrochemical sensor [48].

Porous silicon samples were prepared from p+-type, boron doped silicon wafers with a resistivity of $0.008-0.012 \mathrm{Ohm} \mathrm{cm}$ by standard anodization (electrolyte: $15 \%$ of $\mathrm{HF}$ ) at a current density of $30 \mathrm{~mA} \cdot \mathrm{cm}^{-2}$. The porosity measured by the gravimetrical method was approximately $62 \%$. The pore size was estimated by TEM and ranged from $50 \mathrm{~nm}-75 \mathrm{~nm}$ in diameter. These diameters are large enough to allow the sensing molecules to penetrate and attach. For DNA, the diameter of the nucleotides is approximately $5 \AA$, which is small enough to fit into the porous matrix. Stabilization of $\mathrm{p}-\mathrm{Si}$ is necessaryto passivate its surface and this was done by thermal oxidation. Thermal oxidation of p-Si requires several precautions and high temperatures $\left(>700^{\circ} \mathrm{C}\right)$. Covering the whole internal surface with a thin $\mathrm{SiO}_{2}$ layer stabilizes the structure, permits water penetration into the pores and facilitates probe and target penetration [54]. Al 1 p-Si samples were thermally oxidized in oxygen ambient at $900^{\circ} \mathrm{C}$ for 10 minutes. 
The electrochemical instrumentation used for these experiments included a BAS 100B/W Electrochemical Analyzer and a BAS VC-2 voltammetry cell (model MF-1065). It is well suited for small sizes and has a special micro-cell for volumes as small as $50 \mu \mathrm{L}$. The micro-cell, which included the working electrode, separated a small volume containing the sample from a bulk solution containing the reference and auxiliary electrode with a salt bridge. A platinum wire served as auxiliary electrode and the modified $\mathrm{p}$-Si samples function as working electrodes. It is important to mention that $\mathrm{p}-\mathrm{Si}$, especially oxidized $\mathrm{p}$ - $\mathrm{Si}$, is not conducting and it is in fact the $\mathrm{p}+$ doped silicon that was conducting the electrical current. The top area of the exposed PSi samples was $0.8 \mathrm{~cm}^{2}$ and all lateral areas were insulated with a commercial epoxy resin (see figure 6). The epoxy resin was deposited very carefully and dried for one hour. The samples were attached to the electrochemical system as shown in fig. 6. Potentials were measured relative to an aqueous, saturated $\mathrm{Ag} / \mathrm{AgCl}$ double junction (reference electrode). The voltammetry experiments were carried out at different scan rates in an electrochemical buffer solution composed by $50 \mathrm{mM}$ sodium phosphate ( $\mathrm{pH} 7$ ) with $0.7 \mathrm{M} \mathrm{NaCl}$. A schematic representation of the electrochemical measurement set up and the electrode arrangement is shown in fig. 6 .

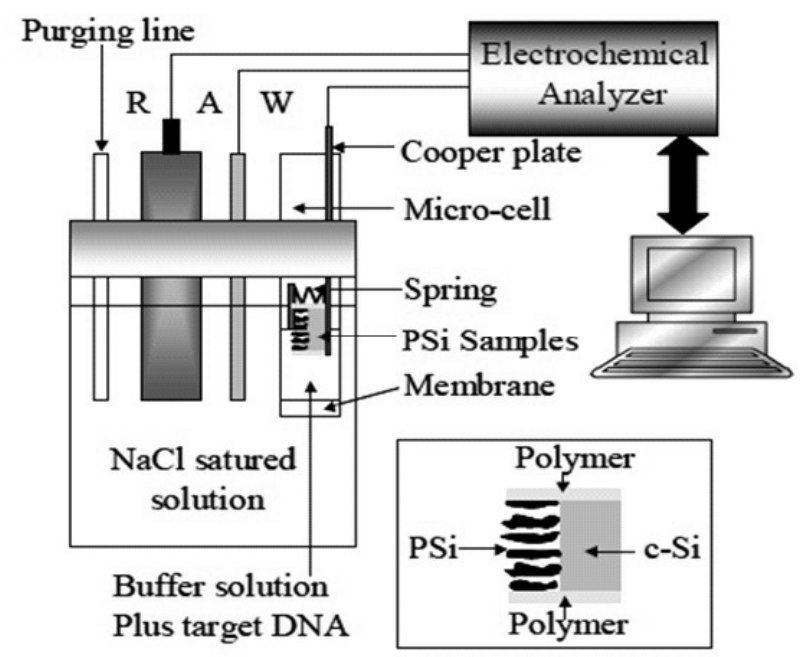

Figure 6. Measurement system: the $\mathrm{p}$-Si electrode is used as working electrode. A platinum wire is the auxiliary electrode and $\mathrm{Ag} / \mathrm{AgCl}$ the reference electrode. Inset: cross section showing the different parts of the working electrode [48].

Three different synthetic oligonucleotides were obtained from MWG Biotech, INC, and have the following sequences: (probe): 5'-TAI-CTA-TII-AAT-TCC-TCI-TAI-ICA-3',(target):5'GCCTAC-GAG-GAA-TTC-CAT-AGC-T-3' and (two-base mismatch target):5'-GCC-TACGAG-GAA-TTG-GAT-AGC-T-3. Tris(2,2'-bypyridyl) ruthenium (II) chloride hexahydrate was purchase from Strem Chemicals. All other chemicals were of analytical grade and purchased from Aldrich and Fluka. Deionized distilled water was obtained from Millipore. 
The detection of DNA consists of the following steps: p-Si silanization, probe immobilization, hybridization, and voltammetric detection.

Several methods may be employed to bind DNA to different supports [55]. One method commonly used for binding DNA involves silanization of an oxidized surface. The function of silane coupling agents is to provide stable bond between two non-bonding surfaces: for example, an inorganic surface to an organic molecule. 3-glycidoxypropyltrimethoxysilane was used to silanize the oxidized p-Si A 5\% aqueous solution of silane was prepared $(\mathrm{pH}$ 4.0). This converts silane into a reactive silanol through hydrolysis. The p-Si samples were then immersed into the continuosly stirred solution and left overnight. 3-glycidoxypropyltrimethoxysilane is hydrolyzed to a reactive silanol by using double distilled water $(\mathrm{pH} 4)$. p-Si samples were then submerged into silanol solution for approximately 17 hours. Constant stirring of the solution was necessary to continuously mix the solution.

After successful silanization, DNA was immobilized onto the surface of p-Si through diffusion. Aqueous solutions of DNA containing $150 \mu \mathrm{l}$ of DNA $(50 \mu \mathrm{M})$ were carefully placed directly above de p-Si layer. The DNA molecules covalently bond to the silanized surface, where they become immobilized. The samples were then placed in a steam container where they were heated in an oven at $37^{\circ} \mathrm{C}$ for approximately 20 hours. The DNA attached samples were then rinsed in double distilled water and dried with nitrogen.

The DNA attached to p-Si was exposed to its complementary strand DNA (target), the mismatch sequence (mismatch probe) and itself (probe). Binding was allowed to proceed for 1 hour at room temperature into hybridization buffer containing $1 \mathrm{M} \mathrm{NaCl}, 10-20 \mathrm{mM}$ sodium cacodylate, $0.5 \mathrm{mM}$ EDTA, $150 \mathrm{mM} \mathrm{KCl}$ and $5 \mathrm{mM} \mathrm{MgCl}$. Throughout the steps, binding was confirmed using Fourier Transform Infrared Spectroscopy (results not shown here).

Cyclic voltammetry (CV) was carried out having the DNA modified, p-Si electrode as working electrode, an $\mathrm{Ag} / \mathrm{AgCl}$ as the reference electrode, and platinum wire as the counter electrode. $6 \mu \mathrm{l}$ of $R u(b p y){ }^{2+}{ }_{3}(0.1 \mu \mathrm{M})$ was poured into $150 \mu \mathrm{l}$ of electrochemical buffer solution. After allowing the solution to diffuse into the samples for 15 minutes, CV was performed. Solutions were deoxygenated via purging with nitrogen for 10 minutes prior to measurements.

p-Si DNA-electrodes and $R u(b p y){ }^{2+}{ }_{3}$ were used for specific gene detection. $R u(b p y){ }^{2+}{ }_{3}$ exhibits a reversible redox couple at $1.05 \mathrm{~V}$ and oxidizes guanine in DNA at high salt concentration [56] according to:

$$
\begin{gathered}
R u(b p y)_{3}^{2+} \rightarrow R u(b p y)_{3}^{3+}+e^{-} \\
R u(b p y)_{3}^{3+}+D N A \rightarrow D N A_{o x}+R u(b p y)_{3}^{2+}
\end{gathered}
$$

where $\mathrm{DNA}_{\mathrm{ox}}$ is a DNA molecule in which guanine has been oxidized by $R u(b p y)^{3+}{ }_{3}$. If the DNA probe contains guanine then $R u(b p y){ }^{3+}{ }_{3}$ will oxidize guanine in DNA, even without the presence of the target DNA. In order to prevent that the DNA probe reacts with $R u(b p y)$ ${ }^{2+}{ }_{3}$ the guanine has been replaced for another less reactive nucleotide. Some previous results 
show that the addition of an oligonucleotide that does not contain guanine produces a small enhancement in the oxidation current [56]. Those results have shown that the inosine $5^{\prime}-$ monophosphate is 3 orders of magnitude less electrochemically reactive than guanosine $5^{\prime}$ monophosphate and still recognizes cytidine [56]. This fact is very important to recognize all four bases in the target sequence. Nevertheless there is a drawback that can have consequences on the hybridization efficiency. Since the deaminated hypoxanthine in the ionosine can only form two of the three hydrogen bonds in a Watson-Crick base pair, it may be desirable to use a guanine derivative that is redox-inert but capable of forming all three hydrogen bonds. However some studies have shown [56] that the specificity afforded by inosine substitution was sufficient but they propose 7-deazaguanine as alternative. For this reason the DNA probe sequence does not contain the guanine base but the target does. Figure 7 (top) shows the CV obtained in solution for the hybrid DNA (probe-target) at different scan rates (target DNA concentration of $0.5 \times 10^{-10} \mathrm{M}$ ). Figure 7 (bottom) shows that the anodic current of $R u(b p y){ }^{2+}{ }_{3}$ is linearly proportional to the scan rate.

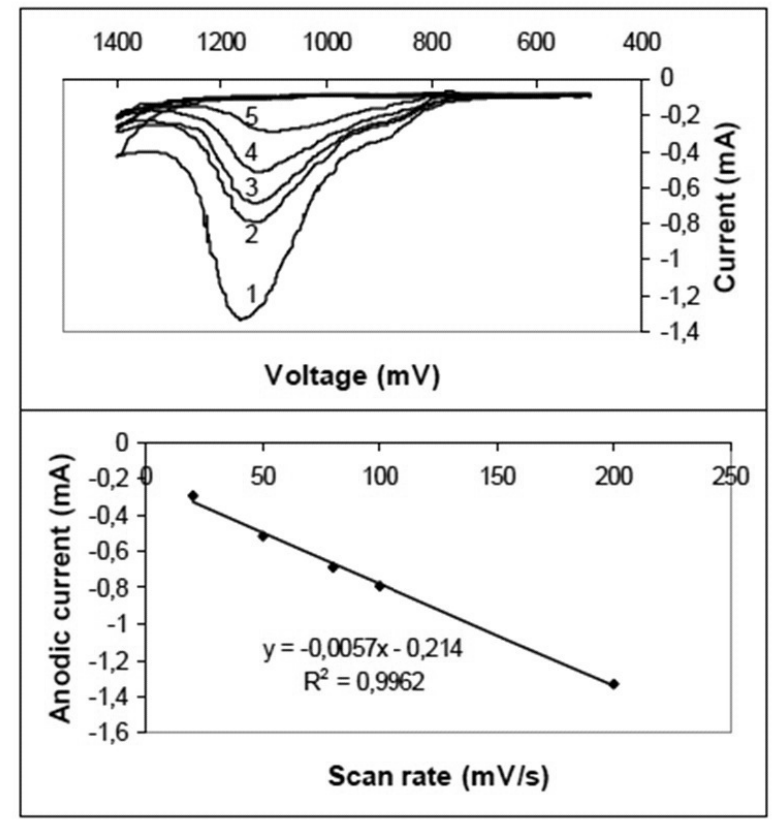

Figure 7. (top) Cyclic voltammograms of the probe-target DNA sequence in $0.1 \mu \mathrm{M} R u(b p y)^{2+}{ }_{3}$ solution at different scan rates (mV.s $\mathrm{s}^{-1}$ ): (1) 20; (2) 50; (3) 80; (4) 100; (5) 200. (bottom) The anodic current changed with the scan rate. The target concentration was $0.5 \times 10^{-10} \mathrm{M}[48]$.

This result is congruent with a process that is controlled by adsorption. Figure 8(top) shows the CV (scan rate of $50 \mathrm{mV} . \mathrm{s}^{-1}$ ) of varied concentrations of target DNA (probe-target sequence, curves 2 to 5) and different targets (probe-mismatch target sequence, curve 1 and 
probe-probe sequence, curve 6). In curve 1, the mismatch target sequence contains two more pairs of base $G$ than the target sequence and that is why the current in this case is bigger than the current obtained in the probe-target sequence cases (curves 2 to 5) or the probeprobe sequence (curve 6). Moreover in curve 6 the current intensity decreases as a consequence of the absence of the

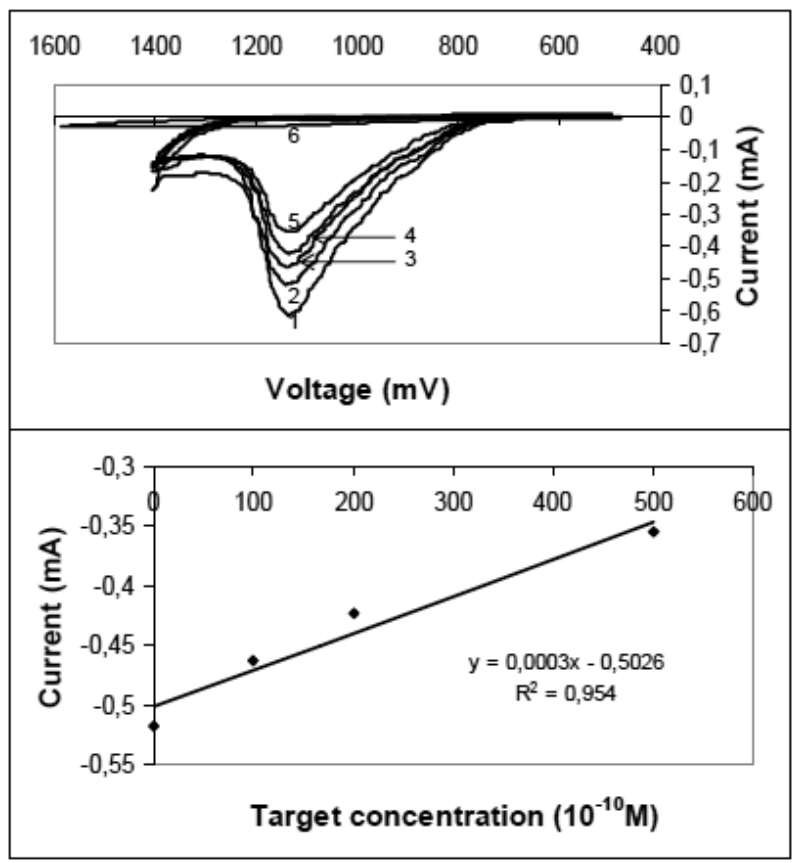

Figure 8. top) Cycled voltammograms of different concentrations of target DNA: (2) $0.5 \times 10^{-10} \mathrm{M}$, (3) $100 \times 10^{-10} \mathrm{M}$, (4) $200 \times 10^{-10} \mathrm{M},(5) 500 \times 10^{-10} \mathrm{M}$. Curve 1 shows the $\mathrm{CV}$ for the probe-mismatch target DNA sequence $\left(0.5 \times 10^{-10} \mathrm{M}\right)$ and curve 6 for the probe-probe DNA sequence $\left(0.5 \times 10^{-10} \mathrm{M}\right)$. (bottom) The anodic current changed with the concentration of the target DNA. In all cases, $0.1 \mu \mathrm{M}$ of $R u(b p y){ }^{2+}{ }_{3}$ was used [48].

base $\mathrm{G}$ in the probe. Nevertheless a significant increase in current was observed for curves 2 to 5 where the target DNA undergoes hybridization to the complementary DNA. This current increase suggests that the hybridization was successful and that the electron transfer from the guanines of the hybridized strand to $R u(b p y){ }^{2+}{ }_{3}$ is responsible for the increase in the current. In comparing curves 1, 2 and 6 (same DNA concentration but different target sequence) it is observed that the sensor responds differently to each target and therefore a good selectivity is achieved. Figure 8 (bottom) shows the anodic peak currents of $R u(b p y)^{2+}{ }_{3}$ at four different concentrations (curves 2 to 5). The peaks are linearly related to the concentration of the target DNA sequence between $0.5 \times 10^{-10}$ and $500 \times 10^{-10} \mathrm{M}$. The detection limit of this approach was $5 \times 10^{-11} \mathrm{M}$. The sensitivity achieved in this work is similar to the one obtained in reference [57], where a sensitivity of $9.0 \times 10^{-11} \mathrm{M}$ was reported for a sensor that 
uses gold substrates instead. In summary those results clearly show that the p-Si sensor shown here has a good selectivity and sensitivity to the target compound, two very important characteristics that a sensor ought to have.

An overview of the requirements for a good performance of a p-Si biosensor was presented and the generalities of the fabrication of different kinds of these biological sensors as well. In the next section we will discuss the new materials, uses and future of porous silicon.

\section{Future of porous silicon biosensors}

Sensors allow our systems and devices to be in relation with the real events that we need to register or control. So, precision (same response to the same stimuli: repeatability) and accuracy (indicating magnitude value as close as possible to the real magnitude of the stimulus to be sensed: minimum absolute error spread) are two main requirements for any sensor when the industry selects a structure type for market use. However, other properties will define the success of a new kind of sensor in the market. These are: technological compatibility with the existing devices, geometric dimension requirements, low noise insertion, ease of adjustment and setup, low power consumption, performance standardization (linear if possible), low thermal or aging characteristic drifts, robustness, reliability, low obsolescence, and very wide field of applications. $\mathrm{p}$-Si is a material that accomplishes all of these requirements with enough margins to think that it will become increasingly popular in the short term. For instance, integrated circuits (IC) are made of crystalline Silicon, which means it is fully compatible for associating a p-Si sensor to any electronic device. The electrochemical technology used to create a p-Si layer does not collide with the IC lithography. The geometric dimensions required to create this type of sensor are sufficiently small to be integrated in an IC. The homogeneity of the porous and its radius control (internal surface density control) as well as its layer stability is improving very fast.

An important factor to take into account in the implementation of a p-Si biosensor is its chemical stability after sample storage. Due to its high superficial area, p-Si based materials tends to be oxidized when are exposed to air ambient conditions. This oxidation plus the addition of other molecules present in the air ambient could modify the biosensor reponse to an analyte after certain amount of time. Pasivation techniques and surface functionalization described before have been proved being successful to prevent or minimize these stability issues. Work has to be done in order to improve the existing methods and assure the reproducibility of p-Si biosensors reponse over a lapse time of years.

Porous silicon has proven to be a succesful material for biosensing applications $[1,58]$. In some cases even femtomolar concentrations in biomolecules was demostrated [1]. The wide range of applications in sensing biological substances include: healt applications [7, 43, 58], virus detection [43], inmunosensors [59, 1], DNA biosensors [58,60], drug delivery [16], biosecurity on food [61], biological warfare agents [62], implantable biosensor technology [58], among others $[12,15,63]$. Some new trends in the fabrication of $\mathrm{p}$-Si biological sensor devices are the use of nanomaterials [54]. The sensitivity and performance of biosensors are be- 
ing improved by using nanomaterials for their construction allowing simple and rapid in vivo analyses [61]. Recently, the plasmonic properties of metal nanoparticles have been used to develop a p-Si biosensor that present Ramman enhacement [64]. Another attractive method for monitoring biomolecular interactions in a highly parallel fashion is the use of microarrays. This p-Si novel porous chip was demonstrated as stable and reproducible, and the fluorescent bioassay reproducibility has been shown [65].

Lately integrated systems of p-Si has been developed [66, 67] e.g a guided mode biosensor based on grating coupled p-Si waveguide [68].

Another type of biosensensing approach that is appearing is an acoustic wave transducer that is coupled with a bioelement e.g an antibody. When the analyte molecules (antigen) get attached to a membrane, the membrane mass changes, resulting in a modification in the resonant frequency of the transducer that can be measured [66]. Rencently p-Si has been proposed as a good material for this kind of biosensors [64].

Notwithsranding the many advantages of p-Si mentioned during this work, several challenges will need to be overcome to be able to make biosensors a viable commercial product. They fall into two main areas: those concerning the fabrication of $\mathrm{p}$-Si for cost effective and robust devices, and those addressing the ability to handle real-world sample matrices such as whole blood [1]. Both are presently the focus of intensive research and it is reasonable to believe that new and exciting developments will occur in a very near future.

\section{Author details}

M. B. de la Mora ${ }^{1}$, M. Ocampo ${ }^{2}$, R. Doti ${ }^{2}$, J. E. Lugo ${ }^{2 *}$ and J. Faubert ${ }^{2}$

*Address all correspondence to: je.lugo.arce@umontreal.ca

1 Instituto de Física. Universidad Nacional Autónoma de México. Circuito de la Investigación Científica Ciudad Universitaria, México

2 Visual Psychophysics and Perception Laboratory, School of Optometry, University of Montreal, Canada

\section{References}

[1] Andrew, Jane., Roman, Dronov., Alastair, Hodges., \& Nicolas, H. Voelcker. (2009). Porous silicon biosensors in the advance. Trends in Biotechnology Review, 27, 230-240.

[2] Bobby, Pejcic., Roland De, Marco., \& Gordon, Parkinson. (2006). The role of biosensors in the detection of emerging infectious diseases. Analyst, 131, 1079-1090. 
[3] Industry Experts. (2012). Biosensors- A Global Market Overview. http://www.reportlinker.com/p0795991/Biosensors-A-Global-Market-Overview.html, accessed June).

[4] Herino, R., Bomchil, G., Barla, K., Bertrand, C., \& Ginoux, J. L. (1987). Porosity and pore size distribution of porous silicon layers. Journal of Electrochemical Society, 134, 1994-2000.

[5] Cullis, A. G., Canham, L. T., \& Calcott, P. D. J. (1997). The structural and luminescence properties of porous silicon. Journal of Applied Physics, 82, 909-965.

[6] Fauchet, P. M., Tsybeskov, L., Peng, C., Duttagupta, S. P., von Behren, J., Kostoulas, Y., Vandyshev, J. M. V., \& Hirschman, K. D. (1995). Light-emitting porous silicon: materials science, properties, and device applications. IEEE Journal of Selected Topics in Quantum Electronics, 1, 1126-1139.

[7] Mathew , Finny P., \& Alocilja , Evangelyn. C. (2005). Porous silicon-based biosensor for pathogen detection. Biosensors and Bioelectronics, 20, 1656-1661.

[8] Ben, A. Jaballah, Hassen, M., Hajji, M., Saadoun, M., Bessais, B., \& Ezzaouia, H. (2005). Chemical vapour etching of silicon and porous silicon: silicon solar cells and micromachining applications. Physica Status Solidi (a), 202, 1606-1610.

[9] Zhipeng, Huang., Nadine, Geyer., Peter, Werner., Johannes de, Boor., \& Ulrich, Gosele. (2011). Metal-Assisted Chemical Etching of Silicon: A Review. Advanced Materials, 23, 285-308.

[10] V'azsonyi, E., Szil'agyi, E., Petrika, P., Horv'atha, Z. E., Lohner, T., Frieda, M., \& Jalsovszky, G. (2001). Porous silicon formation by stain etching. Thin Solid Films, 388, 295-302.

[11] Luca, De Stefano, Rendina, Ivo., Moretti, Luigi., Tundo, Stefania., \& Mario, Andrea. Rossi. (2004). Smart optical sensors for chemical substances based on porous silicon technology. Applied Optics, 43, 1.

[12] Andrea, Salis, Setzu, Susanna., Monduzzi, Maura., \& Mula, Guido. (2011). Porous Silicon-based Electrochemical Biosensors. Biosensors- Emerging Materials and Applications, Chapter InTech., 17, 334-352.

[13] Vincent, G. (1994). Optical properties of porous silicon superlattices. Applied Physics Letters, 64, 2367-2369.

[14] Anthony, P. F. Turner. (2012). Biosensors: Past, Present and Future. http://www.cranfield.ac.uk/health/researchareas/biosensorsdiagnostics/page18795.html, accessed June).

[15] Kristopher, A., Kilian, Till., Bocking, J., \& Gooding, Justin. (2009). The importance of surface chemistry in mesoporous materials: lessons from porous silicon biosensors. Chemical Communications, 630-640. 
[16] Thesis:. (2012). Biocompatibility and biofunctionalization of mesoporous silicon particles. Luis Maria Bimbo. Division of Pharmaceutical Technology Faculty of Pharmacy University of Helsinki Finland.

[17] Chaniotakis, N., \& Sofikiti, N. (2009). Novel semiconductor materials for the development of chemical sensors and biosensors: a review. Analytica Chimica Acta, 615, 1-9.

[18] Chen, Huajie., Hou, Xiaoyuan., Li, Gubo., Zhang, Fulong., Yu, Mingren., \& Wang, Xun. (1996). Passivation of porous silicon by wet thermal oxidation. Journal of Applied Physics, 79, 3282-3285.

[19] Debarge, L., Stoquert, J. P., Slaoui, A., Stalmans, L., \& Poortmans, J. (1998). Rapid thermal oxidation of porous silicon for surface passivation. Materials Science in Semiconductor Processing, 1, 281-286.

[20] Martin-Palma, R. J., Martinez-Duart, J. M., Salonen, J., \& Lehto-P, V. (2008). Effective passivation of porous silicon optical devices by termal carbonization. 103, 083124-083124-4.

[21] Selena, Chan., Yi, Li., Lewis, J. Rothberg, Benjamin, L. Miller, \& Fauchet, Philippe. M. (2001). Nanoscale silicon microcavities for biosensing. Materials Science and Engineering $C, 15,277-282$.

[22] Lin, V. S. Y., Motesharei, K. K., Dancil, P. S., Sailor, M. J., \& Ghadiri, M. R. (1997). A Porous Silicon-Based Optical Interferometric Biosensor. Science, 278, 840-843.

[23] Keiki-Pua, S. Dancil, Douglas, P. Greiner, \& Michael, J. Sailor. (1999). A Porous Silicon Optical Biosensor:Detection of Reversible Binding of IgG to a Protein A-Modified Surface. Journal of American Chemical Society, 121, 7925-7930.

[24] Xuegeng, Li., Yuanqing, He., \& Mark, T. Swihart. (2004). Surface Functionalization of Silicon Nanoparticles Produced by Laser-Driven Pyrolysis of Silane followed by HF$\mathrm{HNO}_{3}$ Etching. Langmuir, 20, 4720-4727.

[25] Sweetman, Martin J., Shearer, Cameron. J., Shapter, Joseph. G., \& Voelcker, Nicolas. H. (2011). Dual silane surface functionalization for the selective attachment of human neuronal cells to porous silicon. Langmuir The Acs Journal Of Surfaces And Colloids, 27, 9497-9503.

[26] Lorraina, N., Hiraouia, M., Guendouza, M., \& Hajia, L. (2011). Functionalization control of porous silicon optical structures using reflectance spectra modeling for biosensing applications. Materials Science and Engineering: B, 176, 1047-1053.

[27] Linford, M. R., \& Chidsey, C. E. D. (1993). Alkyl monolayers covalently bonded to silicon surfaces. Journal of American Chemical Society, 115, 12631-12632.

[28] Schmeltzer, J. M., Lon, J., Porter, A., Stewart, M. P., \& Buriak, J. M. (2002). Hydride Abstraction Initiated Hydrosilylation of Terminal Alkenes and Alkynes on Porous Silicon. Langmuir, 18, 2971-2974. 
[29] Boukherroub, R., Petit, A., Loupy, A. J., Chazalviel, N., \& Ozanam, F. (2003). Microwave-Assisted Chemical Functionalization of Hydrogen- Terminated Porous Silicon Surfaces. Journal of Physica Chemical B, 107, 13459-13462.

[30] Lugo, J. E., Ocampo, M., Doti, R., \& Faubert, J. (2011). Porous Silicon Sensors- from Single Layers to Multilayer Structures . Biosensors- Emerging Materials and Applications Chapter 15, InTech.

[31] Jeffrey, Fortin. (2009). Chapter 2 Transduction Principles. A. Zribi and J. Fortin (eds.), Functional Thin Films and Nanostructures for Sensors, Integrated Analytical Systems, Springer Science + Business Media.

[32] Claudia, Steinem., Andreas, Janshoff., Victor, S., Lin, Y., Volcker, Nicolas H., \& Ghadiri, Reza M. (2004). DNA hybridization-enhanced porous silicon corrosion: mechanistic investigations and prospect for optical interferometric biosensing. Tetrahedron, 60, 11259-11267.

[33] Yuri, L., Bunimovich, Young., Shik, Shin., Woon-Seok, Yeo., Michael, Amori., Gabriel, Kwong., \& Heath, James R. (2006). Quantitative Real-Time Measurements of DNA Hybridization with Alkylated Nonoxidized Silicon Nanowires in Electrolyte Solution. Journal of American Chemical Society, 128, 16323-16331.

[34] Bisi, O., \& Stefano, Ossicini. L. Pavesi. (2000). Porous silicon: a quantum sponge structure for silicon based optoelectronics. Surface Science Reports, 38, 1-126.

[35] Orosco, M. M., Pacholski, C., Miskelly, G. M., \& Sailor, M. J. (2006). Protein- Coated Porous-Silicon Photonic Crystals for Amplified Optical Detection of Protease Activity. Advanced Materials, 18, 1393-1396.

[36] Cheng, L., Anglin, E., Cunin, F., Kim, D., Sailor, M. J., Falkenstein, I., Tammewar, A., \& Freeman, W. R. (2008). Intravitreal properties of porous silicon photonic crystals: a potential self-reporting intraocular drug-delivery vehicle. British Journal of Ophthalmology, 92, 705-711.

[37] Cunin, F., Schmedake, T. A., Link, J. R., Li, Y. Y., Koh, J., Bhatia, S. N., \& Sailor, M. J. (2002). Biomolecular screening with encoded porous-silicon photonic crystals. Nature Materials, 1, 39-41.

[38] Elizabeth, C., Wu, Jennifer. S., Andrew, Lingyun., Cheng, William. R., Freeman, Lindsey., \& Pearson, Michael. J. Sailor. (2011). Real-time monitoring of sustained drug release using the optical properties of porous silicon photonic crystal particles. Biomaterials, 32, 1957-1966.

[39] Dal, L., Negro, C. J., Oton, Z., Gaburro, L., Pavesi, P., Johnson, A., Lagendijk, R., Righini, M. Colocci, \& Wiersma, D. S. (2003). Light transport through the band-edge states of Fibonacci quasicrystals. Physics Review Letters, 90, 1-4.

[40] Starodub, N. F., Shulyak, L. M., Shmyryeva, O. M., Pylipenko, I. V., Pylipenko, L. N., \& Melnichenko, M. M. (2011). Nanostructured Silicon and its Application as the 
Transducer in Immune Biosensors. NATO Science for Peace and Security Series A: Chemistry and Biology, 2, 87-98.

[41] Huimin, Ouyang., Marie, Archer., \& Philippe, M. Fauchet. (2007). Porous Silicon Electrical and Optical Biosensors. Frontiers in Surface Nanophotonics, Springer Series in Optical Sciences, 133.

[42] Girolamo Di, Francia., Vera La, Ferrara., Sonia, Manzo., \& Salvatore, Chiavarini. (2005). Towards a label-free optical porous silicon DNA sensor. Biosensors and Bioelectronics, 21, 661-665.

[43] Andrea, M., Rossi, Lili., Wang, Vytas., \& Reipa, Thomas. E. Murphy. (2007). Porous silicon biosensor for detection of viruses. Biosensors and Bioelectronics, 23, 741-745.

[44] Palestino, G., de la Mora, M. B., del Rio, J. A., Gergely, C., \& Perez, E. (2007). Fluorescence tuning of confined molecules in porous silicon mirrors. Applied Physics Letters, 91, 1219091-3.

[45] Selena, Chan., Yi, Li., Lewis, J., Rothberg, Benjamin. L., \& Miller, Philippe. M. Fauchet. (2001). Nanoscale silicon microcavities for biosensing. Materials Science and Engineering $C, 15,277-282$.

[46] Kirill, Zinoviev., Laura, G., Carrascosa, Jose., Sanchez del, Rio., Borja, Sepulveda., Carlos, Dominguez., \& Lechuga , M. Laura. (2008). Silicon Photonic Biosensors for Lab-on-a-Chip Applications. Advances in Optical Technologies, 1-6.

[47] Marie, Archer., Marc, Christophersen., \& Philippe, M. Fauchet. (2003). Porous Silicon Electrical Biosensors. 737. Material Research Society Symposium Proceedings.

[48] Lugo, J. E., Ocampo, M., Kirk, A. G., Plant, D. V., \& Fauchet, P. M. (2007). Electrochemical Sensing of DNA with Porous Silicon Layers. Journal of New Materials for Electrochemical Systems, 10, 113-116.

[49] Lenward, Seals., James, L., Gole, Laam., Angela, Tse., \& Hesketh, Peter J. (2002). Rapid, reversible, sensitive porous silicon gas sensor. Journal of Applied Physics, 91-94, 2519.

[50] Joon-Jyung, Jin., Se-Hwan, Paek., Chi-Woo, Lee., \& Nam-Ki, Min. (2003). Fabrication of Amperometric Urea Sensor Based on Nano-Porous Silicon Technology. Journal of the Korean Physical Society, 42, S 735-S738.

[51] Marion, Thust. M. J., Schoning, S., Frohnhoff, R., Arens-Fischer, P., \& Luth, Kordos H. (1996). Porous silicon as a substrate material for potentiometric biosensors. Measurement Science and Technology, 7, 26-29.

[52] Joon-Hyung, Jin., Evangelyn, C. Alocilja, \& Grooms, Daniel L. (2010). Fabrication and electroanalytical characterization of label-free DNA sensor based on direct electropolymerization of pyrrole on p-type porous silicon substrates. Journal of Porous Materials, 17, 169-176. 
[53] Min-Jung, Song., Dong-Hwa, Yun., Nam-Ki, Min., \& Suk-In, Hong. (2007). Electrochemical biosensor array for liver diagnosis using silanization technique on nanoporous silicon electrode. Journal of bioscience and bioengineering , 103, 32-37.

[54] Archer, M., \& Fauchet, P. M. (2003). Electrical sensing of DNA hybridization in porous silicon layers. Physics. Status Solidi, 198, 503-508.

[55] Isola, N., Stokes, D. L., \& Vo-Dinh, T. (1998). Surface-Enhanced Raman Gene Probe for HIV Detection". Analytical Chemistry, 70, 1352-1356.

[56] Napier, M. E., Loomis, C. R., Sistare, M. F., Kim, J., Eckhardt, A. E., \& Thorp, H. H. (1997). Biomolecule Recognition with Electron Transfer: Electrochemical Sensors for DNA Hybridization. Bioconjugate Chemical, 8, 906-913.

[57] Yan, F., Erdem, A., Meric, B., Kerman, K., Ozsoz, M., \& Sadik, O. A. (2001). Electrochemical DNA Biosensors for Gene Related Microcystis species. Electrochemical Communications, 3, 224-228.

[58] Chen, Jianrong., Miao, Yuqing., He, Nongyue., Wu, Xiaohua., \& Li, Sijiao. (2004). Nanotechnology and biosensors. Biotechnology Advances, 22, 505-518.

[59] Meskinia, O., Abdelghani, A., Tlili, A., Mgaieth, R., Jaffrezic-Renault, N., \& Martelet, C. (2007). Porous silicon as functionalized material for immunosensor application. Talanta, 71, 1430-1433.

[60] Luca De, Stefano., Paolo, Arcari., Annalisa, Lamberti., Carmen, Sanges., Lucia, Rotiroti., Ilaria, Rea., \& Ivo, Rendina. (2007). DNA Optical Detection Based on Porous Silicon Technology: from Biosensors to Biochip. Sensors, 7, 214-221.

[61] Radke, S. M. (2005). A microfabricated biosensor for detecting foodborne bioterrorism agents. Sensors Journal IEEE, 5, 744-750.

[62] Gooding, Justin J. (2006). Biosensor technology for detecting biological war- fare agents: Recent progress and future trends. Analytica Chimica Acta, 559, 137-151.

[63] Miller, Benjamin L., Amarjeet, S. Bassi, \& Knopf, George K. (2006). Porous Silicon in Biosensing Applications. Smart Biosensor Technology, 271-290.

[64] Yang, Jiao., Dmitry, S., Koktysh, Nsoki., Phambu, Weiss, \& Sharon, M. (2010). Dualmode sensing platform based on colloidal gold functionalized porous silicon. Applied Physics Letters, 97, 1531251-3.

[65] Anton, Ressine., Gyorgy-Varga, Marko., \& Thomas, Laurell. (2007). Porous silicon protein microarray technology and ultra-/superhydrophobic states for improved bioanalytical readout. Biotechnology Annual Review, 13, 149-200.

[66] Mohanty, Saraju P. (2012). Biosensors : A Survey Report. http://biocapteurs.wikispaces.com/file/view/BiosensorSurveyReport.pdfaccessed June) 
[67] Iryna, V., Gavrilchenko, Arthur. I., Benilov, Yuriy. G. Shulimov, \& Skryshevsky, Valeriy A. (2009). Thermally induced acoustic waves in porous silicon. Physica Status Solidi (c), 1725-1728.

[68] Xing, Wei., \& Sharon, M. Weiss. (2011). Guided mode biosensor based on grating coupled porous silicon waveguide. Optics Express, 19, 11330-11339.

[69] Jesus, Alvarez., Paolo, Bettotti., Isaac, Suarez., Neeraj, Kumar., Daniel, Hill., Vladimir, Chirvony., Lorenzo, Pavesi., \& Juan-Pastor, Martinez. (2011). Birefringent porous silicon membranes for optical sensing. Optics Express, 19, 26106-26116. 
